\title{
The Long-Term Impact of High Levels of Alpha-Melanocyte-Stimulating Hormone in Energy Balance Among Obese Adolescents
}

\author{
Ana Claudia Pelissari Kravchychyn ${ }^{a}$ Raquel Munhoz da Silveira Campos ${ }^{b}$ \\ Flávia Campos Corgosinhoc ${ }^{c}$ Deborah Cristina Landi Masquio $^{d}$ \\ Sofia Emanuelle de Castro Ferreira Vicente ${ }^{a}$ Yasmin Alaby Martins Ferreira ${ }^{a}$ \\ Patrícia Leão Silva ${ }^{c}$ Aline de Piano Ganen ${ }^{d}$ Lila Missae Oyama ${ }^{a}$ Lian Tock $^{\mathrm{e}}$ \\ Marco Túlio de Mello ${ }^{f}$ Sérgio Tufik ${ }^{a}$ Ana Raimunda Dâmaso ${ }^{a}$ \\ ${ }^{a}$ Federal University of São Paulo, São Paulo, Brazil; b Federal University of São Carlos, São Paulo, Brazil;

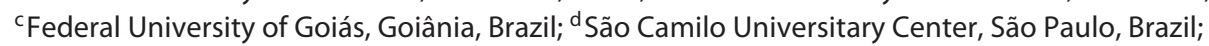 \\ 'Weight Science, São Paulo, Brazil; ${ }^{\text {} F e d e r a l ~ U n i v e r s i t y ~ o f ~ M i n a s ~ G e r a i s, ~ M i n a s ~ G e r a i s, ~ B r a z i l ~}$
}

\section{Keywords}

Energy balance $\cdot$ Obesity · Adolescents

\begin{abstract}
Background: Deregulation of orexigenic and anorexigenic pathways occurs among adolescents with obesity. Alphamelanocyte-stimulating hormone (a-MSH) is a key catabolic mediator of energy homeostasis and an important anorexigenic neuropeptide in the control of energy balance and thermogenesis. However, it was not well explored if a-MSH can modulate long-term weight loss therapy responses in a dependent manner according to its concentration. Our hypothesis is that a high a-MSH concentration at baseline promotes better modulation of anorexigenic/orexigenic pathways in obese adolescents. Methods: One hundred ten post-pubertal obese adolescents (body mass index $>95$ th percentile) were submitted to 1 year of interdisciplinary
\end{abstract}

therapy (clinical, nutritional, psychological, physical exercise, and physiotherapy support). Body composition and plasma levels of a-MSH, neuropeptide $\mathrm{Y}$ (NPY), melaninconcentrating hormone, and agouti-related peptide (AgRP) were measured before and after therapy. The volunteers were grouped on the basis of Tertiles of a-MSH concentration: Low $(<0.75 \mathrm{ng} / \mathrm{mL}$ ), Medium ( $\leq 0.76$ to $\geq 1.57 \mathrm{ng} / \mathrm{mL}$ ), and High ( $>1.57 \mathrm{ng} / \mathrm{mL}$ ). Significance was set as $p<0.05$. Results: The treatment promoted a significant improvement in body adiposity and fat free mass for all groups. It is important to note that only in the high a-MSH group, a significant increase of the a-MSH/NPY ratio and decrease NPY/ AgRP ratio post treatment were observed. Conclusion: The high a-MSH concentration promotes better modulation of anorexigenic/orexigenic pathways in obese adolescents following long-term weight loss therapy and this is important in clinical practice.

(c) 2018 S. Karger AG, Basel

\section{KARGER}

(C) 2018 S. Karger AG, Basel

E-Mail karger@karger.com

www.karger.com/anm
Ana Claudia Pelissari Kravchychyn, MSc and Ana Raimunda Dâmaso, PhD

Post-Graduation Program of Nutrition

Paulista Medicine School, Universidade Federal de São Paulo

Rua Botucatu, 862, Vila Clementino, São Paulo/SP 04024-002 (Brazil)

E-Mail anapelissari@ hotmail.com and ana.damaso@ unifesp.br 


\section{Introduction}

Energy balance is controlled through several physiological signals that regulate food intake in both short- and long-term promoting whole body homeostasis. The body energy is regulated by the arcuate nucleus (ARC) of the hypothalamus through the release of orexigenic and anorexigenic neuropeptides. The release of these neuropeptides is regulated by endocrine and neuronal input, and when this signaling pathway fails, the energy balance is deregulated [1-3].

Neurons expressing these neuropeptides interact with peripheral signals, such as leptin signaling, acting to energy balance pathways. There are neurons that synthesize the anorexigenic neuropeptides cocaine-and-amphetamineregulated transcript and proopiomelanocortin (POMC). When stimulated, POMC releases hypothalamic alphamelanocyte-stimulating hormone ( $\alpha-\mathrm{MSH})$, which is a key catabolic mediator of energy homeostasis. The peptide $\alpha-\mathrm{MSH}$ is a part of the melanocortin (MC) system, which is made up of $\alpha, \beta$, and $\gamma-\mathrm{MSH}$, adrenocorticotropic hormone, MC receptors, agouti-related peptide (AgRP), and endogenous MC receptors antagonist $[2,4]$.

In the ARC, the synthesis of orexigenic neuropeptides, such as neuropeptide Y (NPY) and AgRP, also occurs. These neuropeptides are inhibited by leptin, being antagonists of the $\alpha-\mathrm{MSH}$ and modulating appetite stimulation, lipogenesis, and reducing energy expenditure $[2,4$, 5]. The deregulation in orexigenic and anorexigenic neuropeptides expression is the basis to understanding the positive energy balance and the determinant factor of the increase in adiposity associated with energy intake and low levels of physical activity.

In obesity, the hyperleptinemia state caused by increased adipose tissue may promote a hypothalamic resistance of this hormone action, deregulates anorexigenic neuropeptides expression, as the $\alpha-\mathrm{MSH}$ and contributes to the positive energy balance in obese people $[6,7]$. In a previous study, it was demonstrated that $75 \%$ of obese adolescent present hyperleptinemia, which may impair the weight loss therapy [8].

In this context, negative correlations between body mass insex (BMI) and a-MSH levels were previously demonstrated [9], indicating positive relationship between weight loss and the activation of anorexigenic pathways after long-term weight loss therapy [5]. The a-MSH also plays a role in thermogenesis regulation by mobilizing fat stores and increasing circulating free fatty acids, promoting a favorable environment for negative energy balance [1]. Moreover, recently the $\alpha-\mathrm{MSH}$ was related to the capability of promoting browning effects, thus contributing to our understanding about energy expenditure in human [10].

New insights can suggest an association between a-MSH and its predictive role as a response to weight loss therapy. However, as we know, this hormone it is not fully investigated in obese adolescents undergoing weight loss therapy $[11,12]$. We hypothesized that high concentration of $\alpha-\mathrm{MSH}$ at baseline promotes better modulation of anorexigenic/orexigenic pathways in obese adolescents. Therefore, the present investigation aimed to assess the role of $\alpha-\mathrm{MSH}$ levels on body adiposity and orexigenic/anorexigenic neuropeptides responses in obese adolescents submitted to long-term interdisciplinary therapy. In addition, these data contribute to literature showing the effects of long-term weight loss therapy on the modulation of energy balance pathways.

\section{Methods}

\section{Subjects}

This study involved post pubertal 110 obese adolescents to both gender and age from 14 to 19 years. Inclusion criteria were Tanner stage five [13], primary obesity and BMI $>97$ th percentile of the WHO reference growth charts. Non-inclusion criteria were the use of birth control pills, cortisone, anti-epileptic drugs, and history of renal disease, alcohol intake, smoking, and secondary obesity due endocrine disorders.

The study was conducted according to the principles of the Declaration of Helsinki and was approved by the Ethics Committee on research at the Universidade Federal de Sao Paulo-UNIFESP (\#0135/04), Clinical Trials: NCT01358773. To determine the sample size, G*Power software version 3.1.7 was used. The sample size with 108 volunteers is required to level of significance with $p<$ 0.05 , effect size to $30 \%$, and observed power of $80 \%$ for the analysis of variance (ANOVA) two-way test. Predicting the dropout between 20 and $30 \%$ the treatment was started with 148 participants and was completed with 110 adolescents. The main reasons for the treatment dropout were financial and family problems, followed by school and job opportunities.

\section{Anthropometric Measurements and Body Composition}

The weight was measured on a Filizola scale to the nearest $0.1 \mathrm{~kg}$ and the height was measured to the nearest $0.5 \mathrm{~cm}$ with a wallmounted stadiometer (Sanny, model ES 2030). The BMI was calculated as body weight divided by height squared $\left(\mathrm{wt} / \mathrm{ht}^{2}\right)$. Body composition was measured by air displacement plethysmography in a BOD POD body composition system (version 1.69; Life Measurement Instruments, Concord, CA, USA). For this procedure, the volunteers were instructed to be wearing light clothing and barefoot.

\section{Serum Analysis}

Blood samples were collected after an overnight fast (approximately 8:00 am). a- MSH, melanin-concentrating hormone $(\mathrm{MCH}), \mathrm{AgRP}$, and NPY were measured by enzyme-linked immu- 


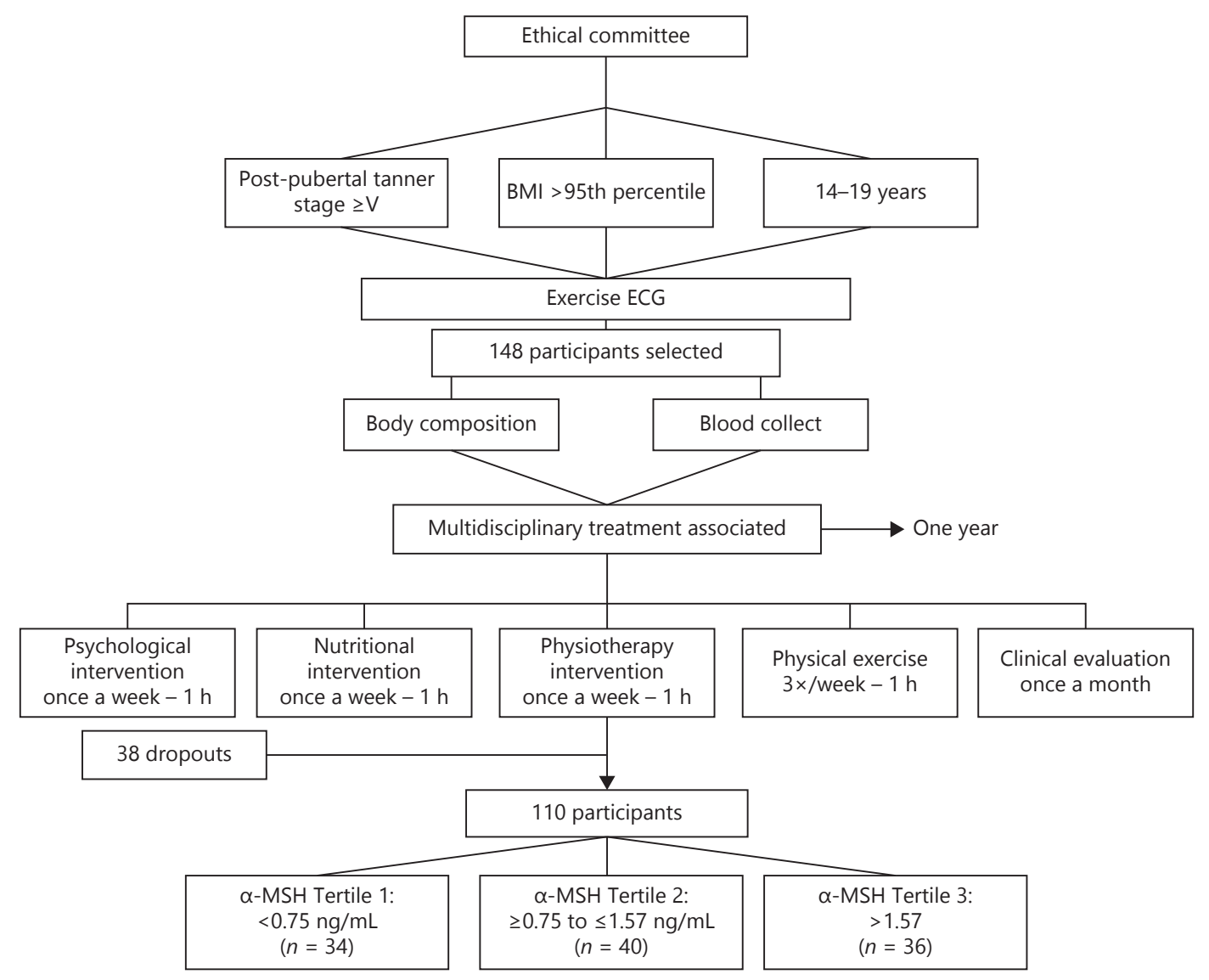

Fig. 1. Diagram of the multidisciplinary therapy. ECG, electrocardiography; $\alpha-M S H$, alpha-melanocyte-stimulating hormone.

nosorbent assay kit from R and D Systems (Minneapolis, MN, USA). Anorexigenic ratio $\alpha-\mathrm{MSH} / \mathrm{NPY}$ and orexigenic ratio NPY/ AgRP were calculated.

\section{Research Design}

The interdisciplinary weight-loss program included clinical, nutritional, psychological physiotherapy, and combined exercise training (aerobic plus resistance training) for change in lifestyle with body weight reduction targets between 5 and $10 \%$. All measurements were performed before and after 1 year of intervention. For statistical analyses, at the end of the study, the volunteers were grouped according to baseline values of $\alpha$-MSH by Tertiles: Low $(<0.75 \mathrm{ng} / \mathrm{mL})$, Medium $(\leq 0.76$ to $\geq 1.57 \mathrm{ng} / \mathrm{mL})$, and High $(>1.57$ ng/mL; Fig, 1).

\section{Clinical Therapy}

During the first visit, the endocrinologist had to determine the inclusion criteria (BMI and post pubertal diagnosis). Medical treatment and follow-up were based on an initial patient and family history, physical examination, and intervention in any health problems that the patient developed over the course of the therapy. The adolescents' clinical support it happened monthly for the assessment of health and clinical parameters.

High Levels of $\alpha$-MSH in Long-Term Weight Loss Therapy

\section{Nutritional Therapy}

The nutritional therapy was conducted by 2 pathways. First, energy intake was set individually for each volunteer and the amount of macronutrients and micronutrients was prescribed according to the Dietary Reference Intake for subjects with low levels of physical activity of the same age and gender. The distribution of macronutrients was between 25 and $35 \%$ for fat, 45 and $65 \%$ for carbohydrate, and 10 and $30 \%$ for protein and some micronutrients such as iron, calcium, vitamin C, and vitamin D, for example, had their intake normalized by including food sources of these nutrients if necessary [14]. Second, once a week, adolescents received dietetics lessons covering the topics related to a healthy eating pattern (example, low-calorie foods, diet and light foods, weight loss diets, good food choices on holidays, weekends and celebrations, food labels, and other related topics). All participants received these 2 interventions. No pharmacotherapies, supplementation, or antioxidant was prescribed.

\section{Psychological Therapy}

The volunteers received weekly group session (1-h). The psychological therapy and the themes talked were conducted based on validated questionnaires considering some of the psychological 
problems caused by obesity, depression, eating disorders, anxiety, decreased self-esteem, and body-image disorders. Individualized psychological therapy was recommended when serious problems were found.

\section{Exercise Protocol}

The exercise program was based on guidelines from the American College of Sports Medicine [15] and was performed 3 times per week and included $30 \mathrm{~min}$ of aerobic training plus 30 min of resistance training per session. All sessions were rigorously supervised by an experienced physiologist. The subjects were instructed to reverse the order of the exercises (aerobic and resistance) at each training session. The aerobic training consisted of running on a motor-driven treadmill (Life Fitness - Model TR $9700 \mathrm{HR}$ ) at a cardiac frequency intensity representing ventilatory threshold 1 ( $\pm 4 \mathrm{bpm})$, according to the results of an initial oxygen uptake test for aerobic exercises. Resistance training was prescribed to main muscle groups and the load of exercise was adjusted after 2 weeks for adaptation to the movement and minimum initial load. Each 8-week, the intensity (load) and volume (repetitions) were adjusted inversely: when decreasing the number of repetitions (from 15-20 to 10-12 and 6-8 repetitions) the load was increased for 3 sets.

\section{Physiotherapy}

The subjects participated in a weekly intervention with 2 physical therapists (1-h). The themes of these group interventions were global postural reeducation, isostretching, diaphragmatic breathing, hydrotherapy, balance, and stretching. Individual assessment was also performed if the patient had any injuries.

\section{Statistical Analysis}

Statistical analysis was performed using STATISTICA software version 7.0 for Windows (StartSoft, Tulsa, OK, USA) with the level of statistical significance set at $p<0.05$. Data normality was verified with the Kolmogorov-Smirnov test. Parametric data were expressed as mean $\pm \mathrm{SD}$, while variables that did not have a normal distribution were normalized by Zscore. Comparisons between genders at baseline were performed by $t$ test and measurements before and after intervention were performed using the ANOVA repeat measures. ANOVA two-way test was applied to compare delta variables among the groups. The test Generalized Linear Model was used with Gama distribution the following variables of interest: $\mathrm{MCH}, \alpha-\mathrm{MSH}, \mathrm{AGPR}, \mathrm{NPY}$, and the $\alpha-\mathrm{MSH} /$ NPY and NPY/AgRP ratio. The choice of distribution considered was based on parsimony between the exploratory analysis of histograms and in the balance good fit Akaike information criterion and Bayesian information criterion. Significance was set as $p<0.05$.

\section{Results}

The study comprised a sample of 110 volunteers grouped according to $\alpha$-MSH levels at baseline: Tertile 1 $(<0.75 \mathrm{ng} / \mathrm{mL} ; n=37)$, Tertile $2(\geq 0.75$ to $\leq 1.57 \mathrm{ng} / \mathrm{mL}$; $n=40)$, and Tertile $3(>1.57 \mathrm{ng} / \mathrm{mL} ; n=36)$.
Table 1. Descriptive data of body composition and energy balance parameters on all volunteers and by gender

\begin{tabular}{lccc}
\hline & All $(n=110)$ & Girls $(n=67)$ & Boys $(n=43)$ \\
\hline Age, years & $16.5 \pm 2.2$ & $16.5 \pm 2.6$ & $16.4 \pm 1.3$ \\
Weight, kg & $107.8 \pm 15.2$ & $100.9 \pm 15.9$ & $108.3 \pm 17.3^{*}$ \\
BMI, kg/m & $37.1 \pm 5.1$ & $37.5 \pm 5.2$ & $34.9 \pm 4.7^{*}$ \\
Body fat, \% & $46.2 \pm 5.0$ & $48.4 \pm 5.3$ & $40.5 \pm 6.6^{*}$ \\
Body fat, kg & $50.1 \pm 10.3$ & $49.0 \pm 11.5$ & $44.5 \pm 12.9$ \\
Free fat mass, \% & $53.8 \pm 5.0$ & $51.7 \pm 5.2$ & $59.5 \pm 6.6^{*}$ \\
Free fat mass, kg & $57.7 \pm 8.1$ & $51.5 \pm 6.6$ & $63.8 \pm 8.2^{*}$ \\
MCH & $2.1 \pm 0.7$ & $5.5 \pm 3.0$ & $5.1 \pm 2.4$ \\
AgRP & $0.3 \pm 0.1$ & $0.8 \pm 0.7$ & $1.0 \pm 1.5$ \\
NPY & $1.0 \pm 0.4$ & $1.0 \pm 0.8$ & $0.9 \pm 0.7$ \\
a-MSH & $0.5 \pm 1.4$ & $1.4 \pm 0.8$ & $1.3 \pm 0.8$ \\
a-MSH/NPY & $0.53 \pm 0.3$ & $0.9 \pm 0.7$ & $0.9 \pm 1.2$
\end{tabular}

Data represented by mean \pm SD.

* Differences between boys and girls at baseline.

AgRP, agouti-related peptide; $\alpha-\mathrm{MSH}$, alpha-melanocyte-stimulating hormone; NPY, neuropeptide Y; $\mathrm{MCH}$, melanin-concentrating hormone.

\section{Descriptive Data and Baseline Values}

Table 1 presents descriptive data for all volunteers and separated by gender in baseline. The comparison showed significant differences in weight, BMI, and percentage of fat and fat-free mass in percentage and in kilograms when the volunteers were separated by gender.

Comparing the baseline data between all 3 groups, there were no significant differences in weight $(\mathrm{kg}), \mathrm{BMI}$ $\left(\mathrm{kg} / \mathrm{m}^{2}\right)$, body fat ( $\%$ and $\left.\mathrm{kg}\right)$, and free fat mass ( $\%$ and $\mathrm{kg})$. Considering the neuropeptides analyses, $\mathbf{a}-\mathrm{MSH}$, NPY, AgRP, and MCH were different between Tertile 3 to 1 and 2, where the Tertile 3 group showed significantly higher values compared with Tertile 1 and 2 groups at baseline. The a-MSH/NPY ratio was significantly higher in the Tertile 2 to Tertile 1 at baseline and the NPY/AgRP ratio was lower in Tertile 2 compared to Tertile 1 (Tables $2,3)$.

\section{Effects of Different Concentrations of $\alpha$-MSH on the} Long-Term Obesity Treatment

The interdisciplinary therapy over 1 year of intervention promoted a significant reduction in body weight $(\mathrm{kg})$, BMI $\left(\mathrm{kg} / \mathrm{m}^{2}\right)$, body fat ( $\%$ and $\left.\mathrm{kg}\right)$, and improvement in free fat mass ( $\%$ and $\mathrm{kg}$ ) in all groups (Table 1). Regarding the neuropeptides, only in the Tertile 3 group, there was a significant change in $\alpha-\mathrm{MSH}, \mathrm{MCH}, \boldsymbol{\alpha}-\mathrm{MSH} /$ NPY ratio, and NPY/AgRP ratio only in Tertile 3 (Tables 2, 3). 


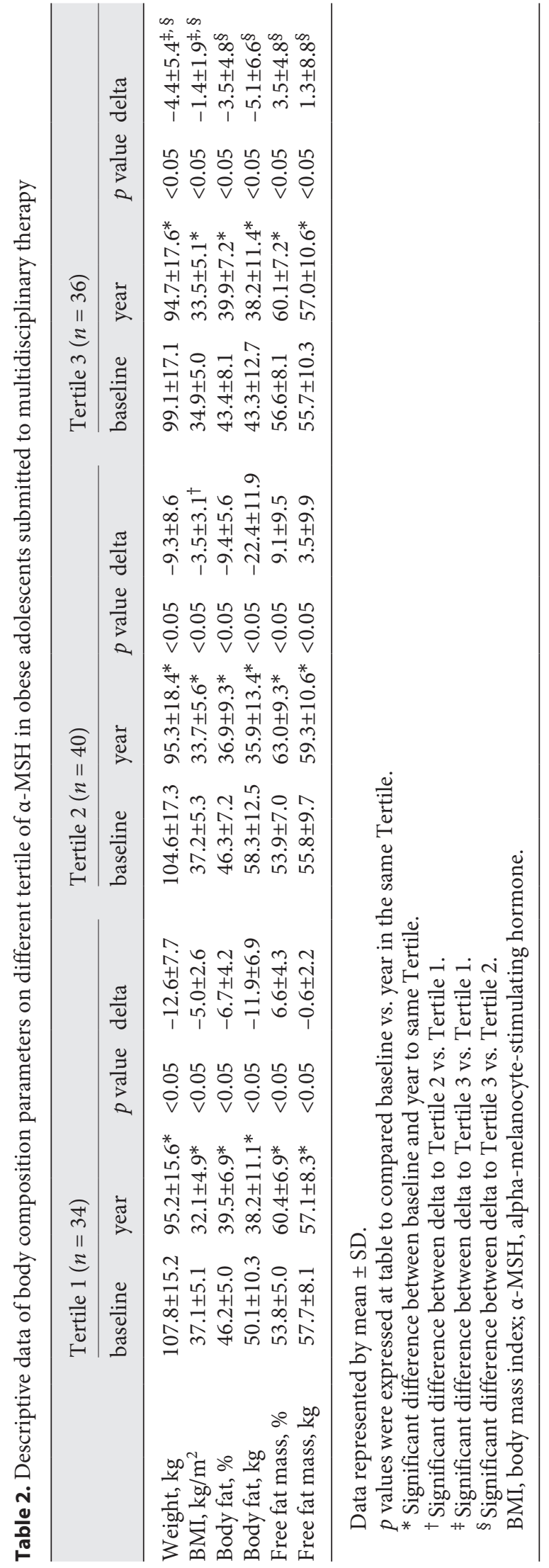

High Levels of $\alpha-\mathrm{MSH}$ in Long-Term Weight Loss Therapy

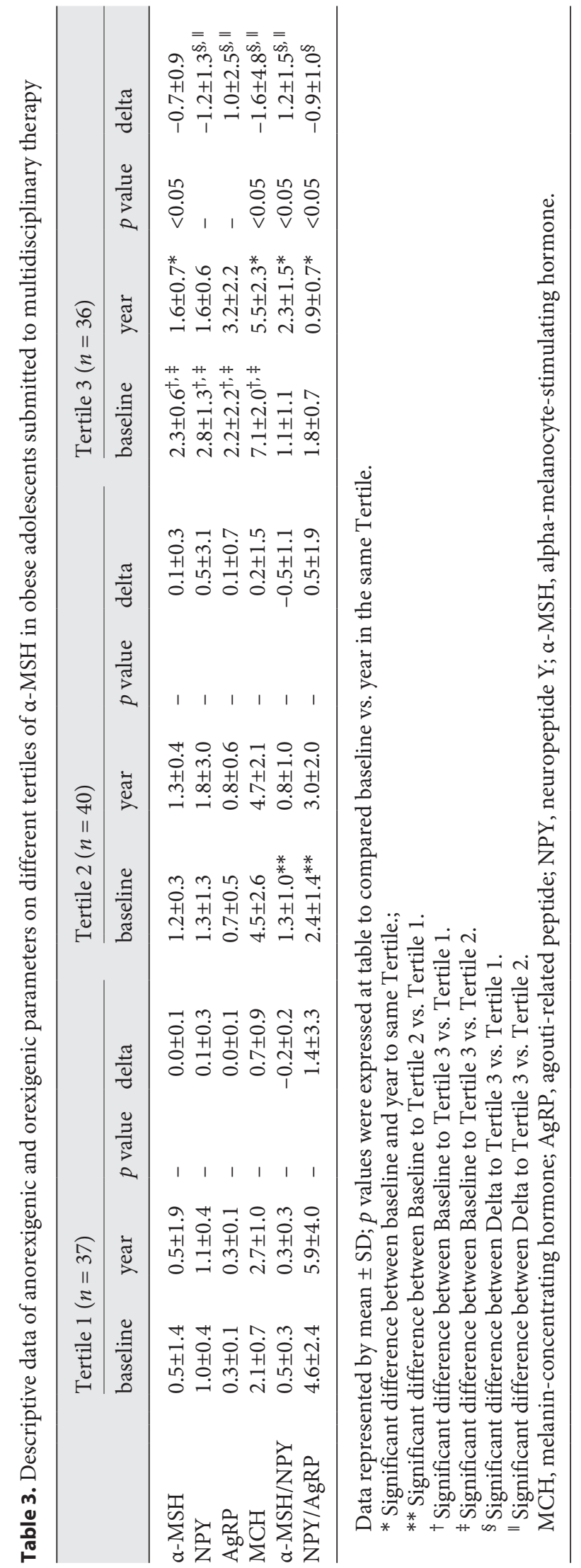

Ann Nutr Metab 2018;72:279-286 
When delta values were analyzed between the groups, a significant difference was observed in body weight and BMI, where Tertile 1 presented higher values of delta. To body fat and free fat mass values, the differences were between Tertile 3 to Tertile 2 and the higher delta values were in Tertile 2 (Table 2 ).

The relationship between $\alpha-\mathrm{MSH}$ levels and orexigenic effects was observed in different delta values of $\mathrm{MCH}$ and $\alpha-\mathrm{MSH} / \mathrm{NPY}$ in all groups. Higher levels of $\alpha-\mathrm{MSH}$ (Tertile3) present more significant changes in the $a-\mathrm{MSH} /$ NPY ratio and MCH compared to Tertiles 1 and 2 and NPY/AgPR ratio compared to Tertile 1 (Table 3).

There were significant statistical differences when comparing the anorexigenic ( $\alpha-\mathrm{MSH})$, orexigenic (NPY, $\mathrm{AgRP}$, and $\mathrm{MCH}$ ), and ratio to anorexigenic ( $\alpha-\mathrm{MSH} /$ NPY) and orexigenic (NPY/AgRP) pathway variables of the 3 groups after treatment. As expected in the Tertile 3 , the $\alpha-\mathrm{MSH} / \mathrm{NPY}$ ratio was significantly higher and the NPY/AgRP ratio was lower compared to Tertiles 1 and 2 (Table 4).

\section{Discussion}

The a-MSH levels appear to be an important key to understand the complex mechanism of energy balance control. In this way, the aim of the present investigation was to assess the role of $\alpha-\mathrm{MSH}$ levels on body adiposity and orexigenic/anorexigenic neuropeptides responses in obese adolescents submitted to long-term interdisciplinary therapy. Interestingly, in the present study, we observed a significant modulation in body composition components, independent of $\alpha$-MSH levels showing the positive effects of weight loss therapy. However, the most important finding of this study was that only when $\alpha-\mathrm{MSH}$ is high in obese patients before treatment, there is a significant modulation of anorexigenic and orexigenic pathways after long-term intervention.

We hypothesized that a high $\alpha$-MSH concentration at baseline promotes better modulation of anorexigenic/orexigenic pathways in obese adolescents. Only in volunteers with high $\alpha$-MSH levels, a significant increase in $a-\mathrm{MSH} / \mathrm{NPY}$ ratio and decrease NPY/AgRP ratio post-treatment was observed, and this showed a possible better modulation of energy balance variables when compared with low and medium a-MSH levels. Even though weight loss occurred in all groups, it was observed that $\mathrm{a}-\mathrm{MSH}$ levels could influence the control of energy balance homeostasis, which can be decisive in
Table 4. Comparative data of anorexigenic and orexigenic parameters post treatment on different Tertiles of $\alpha-\mathrm{MSH}$ in obese adolescents submitted to multidisciplinary therapy

\begin{tabular}{|c|c|c|c|}
\hline & $\begin{array}{l}\text { Tertile } 1 \\
(n=34)\end{array}$ & $\begin{array}{l}\text { Tertile } 2 \\
(n=40)\end{array}$ & $\begin{array}{l}\text { Tertile } 3 \\
(n=36)\end{array}$ \\
\hline \multicolumn{4}{|l|}{ Anorexigenic variable } \\
\hline$\alpha-\mathrm{MSH}$ & $0.5 \pm 0.3$ & $1.2 \pm 0.7^{*}$ & $1.6 \pm 0.9^{*, \dagger}$ \\
\hline \multicolumn{4}{|l|}{ Orexigenic variable } \\
\hline AgRP & $0.3 \pm 0.3$ & $0.8 \pm 0.8^{*}$ & $3.1 \pm 3.4^{*, \dagger}$ \\
\hline NPY & $1.1 \pm 1.2$ & $1.9 \pm 1.9 *$ & $1.6 \pm 1.7^{*}$ \\
\hline $\mathrm{MCH}$ & $2.72 \pm 3.2$ & $4.7 \pm 2.7^{*}$ & $5.5 \pm 3.3^{*}$ \\
\hline \multicolumn{4}{|l|}{ Anorexigenic ratio } \\
\hline$a-\mathrm{MSH} / \mathrm{NPY}$ & $0.3 \pm 0.5$ & $0.8 \pm 1.1^{*}$ & $2.3 \pm 3.2^{*, \dagger}$ \\
\hline \multicolumn{4}{|l|}{ Orexigenic ratio } \\
\hline NPY/AgRP & $5.9 \pm 9.3$ & $3.2 \pm 4.4^{*}$ & $0.9 \pm 1.3^{*, \dagger}$ \\
\hline \multicolumn{4}{|c|}{$\begin{array}{l}\text { Data represented by mean } \pm \text { SD. } \\
\text { * Significant difference to Tertile } 1 \text {. } \\
\text { † Significant difference to Tertile } 2 \text {. } \\
\text { MCH, melanin-concentrating hormone; AgRP, agouti-related } \\
\text { peptide; NPY, neuropeptide Y; } a-M S H \text {, alpha-melanocyte-stimu- } \\
\text { lating hormone. }\end{array}$} \\
\hline
\end{tabular}

maintaining weight post treatment, yo-yo effect prevention, and improve the adherence to a healthy lifestyle post treatment.

To our knowledge, this is the first study to show the behavior of neuroendocrine neuropeptides and body composition variables considering the different concentrations of $\alpha-\mathrm{MSH}$ at baseline. The antagonist relationship between $\alpha-\mathrm{MSH}$ and NPY has been described. In normal conditions, the NPY/AgRP systems are directly stimulated by ghrelin, which leads to an increase in energy intake and acts to depress the activity of $\alpha-\mathrm{MSH}$ in the hypothalamus. In a fed state, the leptin levels suppress NPY and AgRP neurons, stimulate a POMC, and consequently a-MSH $[1,2]$. Besides the function of energy balance control by anorexigenic/orexigenic axis, both neuropeptides also affect the metabolism profile acting in the physiology of adipose tissue: NPY affects the adipogenesis and $\alpha-\mathrm{MSH}$ is responsible for lipolysis [4, $10,16]$.

Composing the MC system, the $\alpha-\mathrm{MSH}$ anorexigenic effects are mediated by specific receptors in the ARC (MC3R and MC4R). Recently, properties other than those that modulate the activity of a broad neural network regulating food intake by suppressing the appetite explain possible influences of the $\mathrm{a}-\mathrm{MSH}$ in the weight loss and reestablishment of balanced neuroendocrine functions. The MC4R and MC5R, receptor of $\alpha-\mathrm{MSH}$ too, have been 
related to the stimulation of metabolic pathways by lipid mobilization and increase in the thermogenesis. This mechanism could occur in the sympathetic nervous system by $\beta$-adrenergic receptors causing the uncoupling of terminal oxidation and ATP production to white adipose tissue and brown adipose tissue [4, 17-20].

The ability of arcuate POMC and AgRP neurons to be regulated by hunger and satiety circulating hormones, including leptin, insulin, ghrelin, estrogens, glucocorticoids, glucagon like peptide 1 , and peptide YY make the MC system sensitive to changes in the nutritional status. For instance, differences in hypothalamic leptin sensitivity presented in obese people (hyperleptinemia state) can influence the stimulation of the $\alpha-\mathrm{MSH}$ hormone and promote failures in satiety by lack of stimulation of anorexigenic pathways $[21,22]$. Since this was a human study, we were not able to clarify more molecular mechanisms, such as $\alpha-\mathrm{MSH}$ receptors sensitivity. However, we believe that this study provides relevant findings to adolescent obesity treatment and contributes to better understanding of human physiology behavior in long-term clinical trials.

In this perspective, Oyama et al. [5] demonstrated how weight loss magnitude can modulate the anorexigenic responses. The a-MSH levels in patients with a small weight loss were reduced significantly; and in massive weight loss, $a$-MSH levels were significantly increased followed by attenuations in NPY and modulations in feeding behavior. Moreover, in the weight loss protocol, the exercise training was suggested as an important tool for modulation to the anorexigenic/orexigenic pathway. In this perspective, Carnier et al. [11] showed how different types of exercises can modulate a-MSH responses, independently of food ingestion, when supported by 1 year of multicomponent weight loss therapy. The aerobic training was able to increase a-MSH levels more than aerobic plus resistance exercise (concurrent training). Despite this result, concurrent exercise in the multidisciplinary context was effective for modulating other important obesity variables involved in metabolic syndrome, nonalcoholic fat liver, bone mineral density, and hyperleptinemia [23-27], thereby justifying why we chose this exercise protocol. It is important to emphasize that despite the results found by Carnier et al. [11], we were able to show improvement in body composition and neuroendocrine modulation with this exercise protocol.

Data that show the behavior of energy balance variables in weight loss therapy contribute to improvements in literature due to the lack of studies about this particular subject. Our study, in which long-term multidisciplinary weight loss therapy was able to reduce weight and BMI, and increase the free fat mass $[9,28]$, brings a new perspective of how anorexigenic neuropeptide levels detection before treatment can be positive to clinical practice. This study presents some limitations: The leptin levels were not found to correlate with $\alpha$-MSH levels, the small sample size post treatment, and lack of a lean control group.

\section{Conclusion}

In summary, the present study showed the impact of different baseline $\alpha$-MSH levels in the modulation of anorexigenic/orexigenic pathways after 1 year of multidisciplinary therapy in obese adolescents. Although the treatment changes body composition in all groups, only in high levels of $\alpha-\mathrm{MSH}$ we could observe significant modulation of the anorexigenic/orexigenic pathway. These results suggest that patients with low levels of a-MSH may have impairments in the regulation of energy balance by pathways of hunger and satiety, which would increase the chances of weight regain and no adherence to post-treatment lifestyle changes.

\section{Acknowledgments}

FAPESP $\quad(2013 / 041364 ; \quad 2013 / 19046-0 ; \quad 2013 / 08522-6$; 2017/07372-1), CNPq (573587/2008-6; 300654/2013-8; 150177/2014-30; 409943/2016-9; 301322/2017-1), and CAPES.

\section{Disclosure Statement}

The authors declare that they have no conflicts of interest to disclose.

References
1 Roh E, Kim MS: Brain regulation of energy metabolism. Endocrinol Metab (Seoul) 2016; 31:519-524.

$\checkmark 2$ Fuqua JS, Rogol AD: Neuroendocrine alterations in the exercising human: implications for energy homeostasis. Metabolism 2013;62: 911-921.

3 Dhillo WS: Appetite regulation: an overview. Thyroid 2007;17:433-445.

4 Shipp SL, Cline MA, Gilbert ER: Recent advances in the understanding of how neuropeptide $Y$ and $\alpha$-melanocyte stimulating hormone function in adipose physiology. Adipocyte 2016;5:333-350. 
5 Oyama LM, do Nascimento CM, Carnier J, de Piano A, Tock L, Sanches Pde L, Gomes FA, Tufik S, de Mello MT, Dâmaso AR: The role of anorexigenic and orexigenic neuropeptides and peripheral signals on quartiles of weight loss in obese adolescents. Neuropeptides 2010;44:467-474.

-6 Hoggard N, Johnstone AM, Faber P, Gibney ER, Elia M, Lobley G, Rayner V, Horgan G, Hunter L, Bashir S, Stubbs RJ: Plasma concentrations of alpha-MSH, AgRP and leptin in lean and obese men and their relationship to differing states of energy balance perturbation. Clin Endocrinol (Oxf) 2004;61:31-39.

7 Dâmaso AR, de Piano A, Sanches PL, Corgosinho F, Tock L, Oyama LM, Tock L, do Nascimento CM, Tufik S, de Mello MT: Hyperleptinemia in obese adolescents deregulates neuropeptides during weight loss. Peptides 2011;32:1384-1391.

$\checkmark 8$ Vehapoğlu A, Türkmen S, Terzioğlu Ş: Alpha-melanocyte-stimulating hormone and agouti-related protein: do they play a role in appetite regulation in childhood obesity? J Clin Res Pediatr Endocrinol 2016;8:40-47.

-9 Dâmaso AR, de Piano A, Campos RM, Corgosinho FC, Siegfried W, Caranti DA, Masquio DC, Carnier J, Sanches Pde L, Leão da Silva P, Nascimento CM, Oyama LM, Dantas AD, de Mello MT, Tufik S, Tock L: Multidisciplinary approach to the treatment of obese adolescents: effects on cardiovascular risk factors, inflammatory profile, and neuroendocrine regulation of energy balance. Int J Endocrinol 2013;2013:541032.

10 Feichtinger RG, Pétervári E, Zopf M, Vidali S, Aminzadeh-Gohari S, Mayr JA, Kofler B, Balaskó M: Effects of alpha-melanocyte-stimulating hormone on mitochondrial energy metabolism in rats of different age-groups. Neuropeptides 2017;64:123-130.

-11 Carnier J, de Mello MT, Ackel-DElia C, Corgosinho FC, Campos RM, Sanches Pde L, Masquio DC, Bueno CR Jr, Ganen Ade P, Martins AC, Caranti DA, Tock L, Clemente AP, Tufik S, Dâmaso AR: Aerobic training (AT) is more effective than aerobic plus resistance training $(\mathrm{AT}+\mathrm{RT})$ to improve anorexigenic/orexigenic factors in obese adolescents. Appetite 2013;69:168-173.
12 Cook CM, Schoeller DA: Physical activity and weight control: conflicting findings. Curr Opin Clin Nutr Metab Care 2011;14:419-424.

13 Tanner JM, Whitehouse RH: Clinical longitudinal standards for height, weight, height velocity, weight velocity, and stages of puberty. Arch Dis Child 1976;51:170-179.

14 NCR. Dietary Reference Intake: Applications in Dietary Assessment. Washington, National Academic Press, 2001.

15 Donnelly JE, Blair SN, Jakicic JM, Manore MM, Rankin JW, Smith BK; American College of Sports Medicine: American college of sports medicine position stand. Appropriate physical activity intervention strategies for weight loss and prevention of weight regain for adults. Med Sci Sports Exerc 2009;41:459-471.

16 Rostás I, Füredi N, Tenk J, Mikó A, Solymár M, Soós S, Székely M, Pétervári E, Balaskó M: Age-related alterations in the central thermoregulatory responsiveness to alpha-MSH. J Therm Biol 2015;49-50:9-15.

17 Mountjoy KG: Distribution and function of melanocortin receptors within the brain. Adv Exp Med Biol 2010;681:29-48.

18 Rodrigues AR, Almeida H, Gouveia AM: Alpha-MSH signalling via melanocortin 5 receptor promotes lipolysis and impairs re-esterification in adipocytes. Biochim Biophys Acta 2013;1831:1267-1275.

19 Richard D, Monge-Roffarello B, Chechi K, Labbé SM, Turcotte EE: Control and physiological determinants of sympathetically mediated brown adipose tissue thermogenesis. Front Endocrinol (Lausanne) 2012;3:36.

20 Song CK, Vaughan $\mathrm{CH}$, Keen-Rhinehart E, Harris RB, Richard D, Bartness TJ: Melanocortin-4 receptor mRNA expressed in sympathetic outflow neurons to brown adipose tissue: neuroanatomical and functional evidence. Am J Physiol Regul Integr Comp Physiol 2008;295:R417-R428.

21 Soos S, Petervari E, Szekely M, Jech-Mihalffy A, Balasko M: Complex catabolic effects of central alpha-MSH infusion in rats of altered nutritional states: differences from leptin. J Mol Neurosci 2011;43:209-216.

22 Nuzzaci D, Laderrière A, Lemoine A, Nédélec E, Pénicaud L, Rigault C, Benani A: Plasticity of the melanocortin system: determinants and possible consequences on food intake. Front Endocrinol (Lausanne) 2015;14:143.

23 Ackel-D'Elia C, Campos RMS, Corgosinho FC, Masquio DCL, Clemente APG, Tock L, de Mello MT, Tufik S, Dâmaso AR: Aerobic plus resistance training on pro-anti-inflammatory adipokinesin obese adolescents. Eur J Sport Exe Sci 2014;3:5-17.

24 Campos RM, de Mello MT, Tock L, Silva PL, Masquio DC, de Piano A, Sanches PL, Carnier J, Corgosinho FC, Foschini D, Tufik S, Dâmaso AR: Aerobic plus resistance training improves bone metabolism and inflammation in adolescents who are obese. J Strength Cond Res 2014;28:758-766.

25 Sanches PL, de Mello MT, Elias N, Fonseca FA, Campos RM, Carnier J, de Piano A, Masquio DC, Silva PL, Oyama LM, Corgosinho FC, Nascimento CM, Tock L, D'Elia CA, Tufik S, Dâmaso AR: Hyperleptinemia: implications on the inflammatory state and vascular protection in obese adolescents submitted to an interdisciplinary therapy. Inflammation 2014;37:35-43.

-26 Dâmaso AR, da Silveira Campos RM, Caranti DA, de Piano A, Fisberg M, Foschini D, de Lima Sanches P, Tock L, Lederman HM, Tufik S, de Mello MT: Aerobic plus resistance training was more effective in improving the visceral adiposity, metabolic profile and inflammatory markers than aerobic training in obese adolescents. J Sports Sci 2014;32:14351445.

27 de Piano A, de Mello MT, Sanches Pde L, da Silva PL, Campos RM, Carnier J, Corgosinho F, Foschini D, Masquio DL, Tock L, Oyama LM, do Nascimento CM, Tufik S, Dâmaso AR: Long-term effects of aerobic plus resistance training on the adipokines and neuropeptides in nonalcoholic fatty liver disease obese adolescents. Eur J Gastroenterol Hepatol 2012;24:1313-1324.

28 Masquio DC, de Piano A, Campos RM, Sanches PL, Carnier J, Corgosinho FC, Netto BD, Carvalho-Ferreira JP, Oyama LM, Nascimento CM, de Mello MT, Tufik S, Dâmaso AR: The role of multicomponent therapy in the metabolic syndrome, inflammation and cardiovascular risk in obese adolescents. $\mathrm{Br} \mathrm{J}$ Nutr 2015;113:1920-1930. 